\title{
ON THE PERFORMANCE OF ITERATIVE DEMAPPING AND DECODING TECHNIQUES OVER QUASI-STATIC FADING CHANNELS
}

\author{
W. R. Carson, I. Chatzigeorgiou and I. J. Wassell \\ Computer Laboratory \\ University of Cambridge \\ United Kingdom
}

\author{
M. R. D. Rodrigues \\ Department of Computer Science \\ University of Porto \\ Portugal
}

\author{
R. Carrasco \\ School of EE\&C Eng. \\ University of Newcastle \\ United Kingdom
}

\begin{abstract}
In this paper, we investigate in detail the performance of iterative demapping and decoding techniques over quasi-static fading channels both with and without antenna diversity. In particular, we consider the effect on the system performance of various mapping schemes, different coding schemes, the interleaver size as well as space diversity. Results demonstrate that over quasi-static fading channels characterized by significant antenna diversity, mappings traditionally optimized for iterative receivers (e.g., Boronka mapping) outperform mappings more appropriate for non-iterative receivers (e.g., Gray mapping). In contrast, over quasi-static fading channels characterized by limited antenna diversity, Gray mapping always outperform Boronka mapping for all $E_{b} / N_{0}$ and all iterations. Strikingly, this situation is in sharp contrast to that in the AWGN case. We note that we can further improve the performance of non-iterative systems (e.g., Gray mapping) having limited antenna diversity by increasing the memory size and removing the interleaving.
\end{abstract}

\section{INTRODUCTION}

The turbo principle was conceived over a decade ago by Berrou et al. [1][2] In particular, the authors proposed an encoder consisting of the parallel concatenation of two recursive systematic convolutional (RSC) encoders separated by a pseudorandom interleaver, and the corresponding turbo decoder consisting of two component soft-input soft-output decoders that exchange soft information in an iterative manner. Turbo codes were shown to exhibit an impressive performance, closely approaching the Shannon limit.

Turbo or iterative techniques have since been proposed for a large number of communication scenarios including multi-user processing [3], space-time processing [4] and the processing of bit interleaved coded modulation (BICM) [5][6].

Iterative techniques have been shown to exhibit improved performance over the additive white Gaussian noise (AWGN) channel [1][2]. In particular, it has been demonstrated that different system parameters, e.g., the interleaver size and the constituent RSC codes in the case of turbo codes, can dramatically affect the performance of iterative techniques over AWGN channels. Moreover, it has been also shown that turbo techniques perform very well over fast fading channels [7]. However, it was shown that turbo techniques perform poorly over slow fading channels [8]. Essentially, over rapidly fading channels interleaving can be used as a means to create diversity by spreading the transmit symbols over multiple independently fading blocks as a means to enhance performance. How- ever, over slow fading channels interleaving cannot be used as a means to create diversity owing to delay and latency considerations. This situation compromises in particular the performance of turbo techniques because occasional deep fades will affect the entire transmit frame causing severe error propagation in the iterative receiver [9].

To date little is known about the performance of iterative techniques over quasi-static fading channels. Bouzekri et al. [10] as well as Rodrigues et al. [11] have studied the effect of quasi-static fading channels on the performance of turbo codes. In particular, the authors have shown that, in contrast to the situation in the AWGN channel, different turbo code parameters including the interleaver size and the constituent RSC codes do not greatly affect the performance of turbo codes in these scenarios. However, the effect of the quasi-static fading channel on other iterative techniques and systems has not been thoroughly studied. Yet, the quasi-static fading situation is extremely important because it models various practical scenarios characterized by extremely low time and frequency diversity, e.g., fixed wireless access (FWA) channels [12].

This paper investigates in detail the performance of the iterative demapping and decoding schemes proposed by ten Brink [5][6] over quasi-static fading channels both with and without antenna diversity. Section II introduces the system model. Section III studies the performance of these iterative schemes in the quasi-static fading scenario. In particular, it considers the effect on the system performance of various mapping schemes, different coding schemes, the interleaver size as well as space diversity. Finally, the main conclusions of this work are summarized in Section IV.

\section{SYSTEM MODEL}

Fig. 1 depicts the communications system model. We consider both single antenna systems $\left(N_{T}=N_{R}=1\right)$ often known as single-input single-output (SISO), which do not exploit space diversity, as well as multiple antenna systems $\left(N_{T}, N_{R}>1\right)$, which do exploit space diversity. The transmitter consists of four main stages: the encoder, the interleaver, the mapper and the space-time processor (see Fig.1). Initially, the information bits are convolutionally encoded, these coded bits are then pseudo-random interleaved. Finally, groups of $\log _{2} M$ interleaved coded bits are mapped to a complex symbol from a unit power $M$-ary quadrature amplitude modulation (QAM) constellation.

In single transmit antenna systems $\left(N_{T}=1\right)$, the space-time processing block does not further process the mapped symbols; instead, the mapped symbols are directly sent to the transmis- 

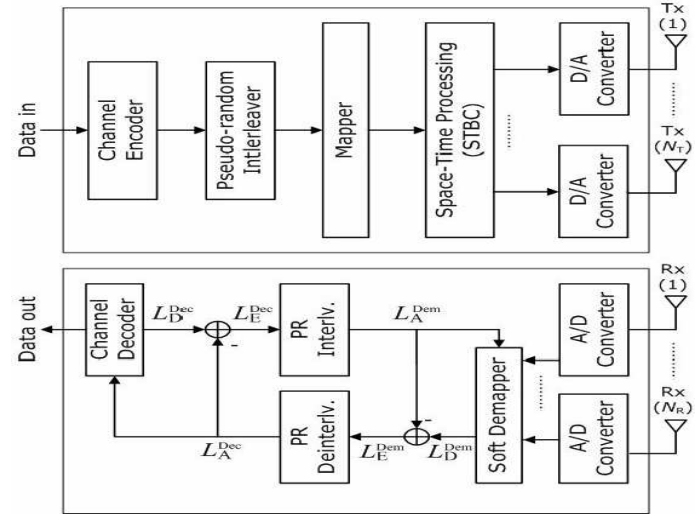

Figure 1: Communications system model.

sion block. However, in multiple transmit antenna systems $\left(N_{T}>1\right)$, the space-time processing block will further process the mapped symbols. In particular, the space-time processor generates a space-time block code (STBC) according to the generator matrices $\mathbf{G}_{2}, \mathbf{G}_{3}$ or $\mathbf{G}_{4}$ given by [13][14]. Essentially, a total of $K \times N_{T}$ symbols obtained from the original $K^{\prime}$ modulation symbols are transmitted during $K$ time slots by $N_{T}$ transmit antennas. Note that $\mathbf{G}_{2}, \mathbf{G}_{3}$ or $\mathbf{G}_{4}$ are appropriate for two, three and four transmit antennas, respectively, and for an arbitrary number of receive antennas. Note also that $\mathbf{G}_{2}$ is rate $\frac{K^{\prime}}{K}=1$, whereas $\mathbf{G}_{3}$ and $\mathbf{G}_{4}$ are rate $\frac{K^{\prime}}{K}=\frac{1}{2}$.

The signal is distorted by a frequency-flat quasi-static fading channel as well as AWGN. Consequently, the relationship between the complex receive symbols and the complex transmit symbols associated with a specific STBC frame may be written as follows ${ }^{1}$ :

$$
\mathbf{r}=\mathbf{h s}+\mathbf{n}
$$

Here, $\mathbf{r}$ denotes the $N_{R}$ by $K$ matrix whose element $r_{j}(k)$ denotes the complex receive symbol at time slot $k$ and receive antenna $j$; s denotes the $N_{T}$ by $K$ matrix whose element $s_{i}(k)$ denotes the complex transmit symbol at time slot $k$ and transmit antenna $i$; $\mathbf{h}$ denotes the $N_{R}$ by $N_{T}$ matrix of channel gains whose element $h_{j, i}$ denotes the channel gain from transmit antenna $i$ to receive antenna $j$ (note that $h_{j, i}$ is independent of time slot $k$ ); and $\mathbf{n}$ denotes the $N_{R}$ by $K$ matrix whose element $n_{j}(k)$ denotes the noise random variable at time slot $k$ and receive antenna $j$. The channel gains are uncorrelated circularly symmetric complex Gaussian with mean zero and variance $\frac{1}{2}$ per dimension. The noise random variables are uncorrelated circularly symmetric complex Gaussian with mean zero and variance $\frac{1}{2 \cdot S N R_{\text {norm }}}=\frac{N_{T}}{2 \cdot S N R}$ per dimension, where SNR denotes the average signal-to-noise ratio per receive antenna.

The receiver consists mainly of two stages: (i) the soft demapper and (ii) the soft-in soft-out decoder, which are separated by pseudo-random interleavers and de-interleavers. These two stages exchange soft information in an iterative manner (see Fig.1). Specifically, the soft demapper takes as a priori information $L_{A}^{D e m}\left(b_{m}(k)\right)$ on the code bits which is an inter-

\footnotetext{
${ }^{1}$ Here, we focus without loss of generality on the first space-time block codes frame
}

leaved version of the extrinsic information $L_{E}^{\text {Dem }}\left(b_{m}(k)\right)$ on the code bits produced by the soft input-soft output decoder. Then, it computes the a posteriori information $L_{D}^{D e m}\left(b_{m}(k) \mid \mathbf{r}\right)$ of the code bits - a log-likelihood ratio (LLR) - given by ${ }^{2}$ :

$$
\begin{gathered}
L_{D}^{\text {dem. }} \cdot\left(b_{m}(k) \mid \mathbf{r}\right)=\ln \frac{\operatorname{Pr}\left(b_{m}(k)=1 \mid \mathbf{r}\right)}{\operatorname{Pr}\left(b_{m}(k)=0 \mid \mathbf{r}\right)}=\ln \frac{\sum_{\left(\mathbf{s} \in \mathbf{s}^{+}\right)} p(\mathbf{r} \mid \mathbf{s}) \operatorname{Pr}(\mathbf{s})}{\sum_{\left(\mathbf{s} \in \mathbf{s}^{-}\right)} p(\mathbf{r} \mid \mathbf{s}) \operatorname{Pr}(\mathbf{s})} \\
=\ln \frac{\operatorname{Pr}\left(b_{m}(k)=1\right)}{\operatorname{Pr}\left(b_{m}(k)=0\right)}+\ln \frac{\sum_{\left(\mathbf{s} \in \mathbf{s}^{+}\right)} p(\mathbf{r} \mid \mathbf{s}) \prod_{\substack{m^{\prime}=1 \\
m^{\prime} \neq m}}^{\log _{2} M} \prod_{\substack{k^{\prime}=1 \\
k^{\prime} \neq k}}^{K^{\prime}} \operatorname{Pr}\left(b_{m^{\prime}}\left(k^{\prime}\right)\right)}{\sum_{\left(\mathbf{s} \in \mathbf{s}^{-}\right)} p(\mathbf{r} \mid \mathbf{s}) \prod_{\substack{\log _{2} M \\
m^{\prime}=1 \\
m^{\prime} \neq m}}^{K^{\prime} \neq} \prod_{\substack{k^{\prime}=1 \\
k^{\prime} \neq k}} \operatorname{Pr}\left(b_{m^{\prime}}\left(k^{\prime}\right)\right)}
\end{gathered}
$$

where $b_{m}(k)$ is the $m$ th bit conveyed by the $k$ th mapped symbol, $\mathbf{s}^{+}$is the set of matrices of transmit symbols $\mathbf{s}$ such that $b_{m}(k)=1$ (i.e., $\left.\mathbf{s}^{+}=\left\{\mathbf{s}: b_{m}(k)=1\right\}\right), \mathbf{s}^{-}$is the set of matrices of transmit symbols s such that $b_{m}(k)=0$ (i.e. $\mathbf{s}^{-}=$ $\left.\left\{\mathbf{s}: b_{m}(k)=0\right\}\right)$, and the probability density function $p(\mathbf{r} \mid \mathbf{s})$ is given by:

$$
p(\mathbf{r} \mid \mathbf{s})=\frac{1}{\left(\pi \cdot S N R_{\text {norm }}^{-1}\right)^{K N_{R}}} e^{-\frac{\operatorname{Tr}\left((\mathbf{r}-\mathbf{h s})^{H}(\mathbf{r}-\mathbf{h s})\right)}{S N R_{\text {norm }}^{-1}}}
$$

Finally, the soft demapper passes the extrinsic information $L_{E}^{D e m}\left(b_{m}(k)\right)=L_{D}^{D e m}\left(b_{m}(k) \mid \mathbf{r}\right)-L_{A}^{D e m}\left(b_{m}(k)\right)$ on the code bits to the subsequent stage - the soft input-soft output decoder.

Likewise, the soft-input soft-output channel decoder takes as a priori information a de-interleaved version of the extrinsic information produced by the soft demapper. Then, it computes the a posteriori information, using a soft input-soft output decoding algorithm, e.g., the SOVA algorithm [15]. Finally, the soft input-soft output decoder passes the extrinsic information back to the subsequent stage - the soft demapper.

In the very first iteration stage, the a priori information $L_{A}^{D e m}\left(b_{m}(k)\right)$ is zero so that the probabilities in (2) are $\operatorname{Pr}\left(b_{m}(k)=1\right)=\frac{1}{2}$ and $\operatorname{Pr}\left(b_{m}(k)=0\right)=\frac{1}{2}$. In subsequent iteration stages $L_{A}^{D e m}\left(b_{m}(k)\right)$ is non-zero so that the probabilities in (2) are $\operatorname{Pr}\left(b_{m}(k)=1\right)=\frac{1}{1+e^{+L_{A}^{D e m}\left(b_{m}(k)\right)}}$ and $\operatorname{Pr}\left(b_{m}(k)=0\right)=\frac{1}{1+e^{-L_{A}^{D e m}\left(b_{m}(k)\right)}}$.

\section{Simulation Results}

This section investigates the performance of iterative demapping and decoding techniques over frequency-flat quasistatic fading channels both with and without antenna diversity. We consider various mapping schemes including 16-QAM with Gray, anti-Gray or Boronka mappings (see Fig.2). We also consider various coding schemes including rate $1 / 2$ RSC codes with octal generator polynomials $(1,5 / 7)$ or $(1,21 / 37)$. Finally, we take into account different interleaver sizes, specifically 2048 and 8192. We will emphasize differences in performance in the quasi-static fading and the AWGN regimes. In our simulations, we will consider specifically a soft input-soft output decoder implementing the SOVA algorithm.

\footnotetext{
${ }^{2}$ This LLR can be simplified further owing to the orthogonal properties of $\mathbf{G}_{2}, \mathbf{G}_{3}$ or $\mathbf{G}_{4}[13][14]$
} 


\begin{tabular}{|c|c|c|c|}
\hline $\begin{array}{l}\text { o111 } \\
1110\end{array}$ & $\begin{array}{l}\text { oo11 } \\
\text { oo1 } \\
1101\end{array}$ & $\begin{array}{l}1011 \\
111 \\
\text { oooo }\end{array}$ & $\begin{array}{l}1111 \\
\text { oboo } \\
1000\end{array}$ \\
\hline $\begin{array}{l}8110 \\
\text { o18o } \\
0100\end{array}$ & $\begin{array}{l}0010 \\
1010 \\
1010\end{array}$ & $\begin{array}{l}1010 \\
0110 \\
0011\end{array}$ & $\begin{array}{l}11 \\
10 \\
01\end{array}$ \\
\hline $\begin{array}{l}\text { o1 } 100 \\
1019 \\
011\end{array}$ & $\begin{array}{l}\text { Oooo } \\
\text { o11 } \\
1000\end{array}$ & $\begin{array}{l}1000 \\
1000 \\
0110\end{array}$ & $\begin{array}{l}\text { o1 } \\
111\end{array}$ \\
\hline $\begin{array}{l}010 \\
010 \\
0101\end{array}$ & & & \\
\hline
\end{tabular}

Figure 2: 16-QAM mapping schemes: Gray (top), anti-Gray (middle) and Boronka (bottom) mappings

\section{A. Effect of Mapping Schemes}

We first assess the effect of different mapping schemes on the performance of iterative demapping and decoding techniques over quasi-static fading channels. Fig. 3 depicts the BER versus $E_{b} / N_{0}$ performance curves for Gray and Boronka 16-QAM mappings and an RSC with generator polynomial $(1,5 / 7)$ in various channel settings. We observe that for all plots, with the exception of Gray mapping, increasing the number of iterations actually decreases performance at low $E_{b} / N_{0}$, but improves BER performance at medium to high $E_{b} / N_{0}$. This relative change in performance is particularly apparent in an AWGN channel for Boronka mapping. We note that as expected, an increase in the number of receiver iterations results in considerable performance improvements for Boronka mapping based schemes [16], but it does not result in any improvement in performance for schemes based on Gray mapping.

We also observe that Boronka mapping schemes outperform Gray mapping schemes in systems with significant antenna diversity for medium to high $E_{b} / N_{0}$. However, these mappings do not outperform the Gray one in systems with limited antenna diversity for all $E_{b} / N_{0}$. Strikingly, this situation over the quasi-static fading case is in sharp contrast to that over the AWGN case, where Boronka mapping outperforms Gray mapping for a large number of receiver iterations and for medium to high $E_{b} / N_{0}$.

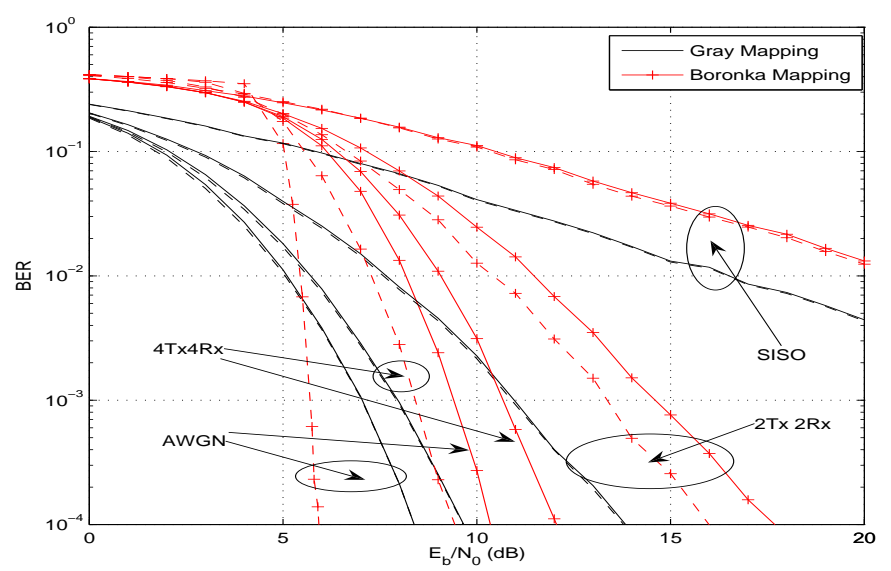

Figure 3: BER versus $E_{b} / N_{0}$ performance curves for iterative demapping and decoding systems based on 16-QAM mappings in AWGN and various quasi-static channel settings. The RSC code generator polynomial is $(1,5 / 7)$. The interleaver size is 2048. Solid lines correspond to no iterations, dashed lines to 20 iterations.
Note that the error rate over a quasi-static fading channel is the average over every possible fading realization of the error rate over an AWGN channel. Consequently, the error rate performance in a quasi-static fading channel with low space diversity - where error rates tend to be high - depends mainly on the behavior of the error probability over AWGN channels in the low to medium $E_{b} / N_{0}$ regime. In turn, the error rate performance over quasi-static fading channels with high space diversity - where error rates tend to be low - depends on the behavior of the error rate over AWGN channels both in the low and high $E_{b} / N_{0}$ regime. This explains the difference in behavior between the different system settings since it turns out that the probability of error for Gray mapping is lower than that for Boronka mappings in the low to medium $E_{b} / N_{0}$ regime, and higher for medium to high $E_{b} / N_{0}$.

\section{B. Effect of Coding Schemes}

We now assess the effect of different coding schemes on the performance of iterative demapping and decoding techniques over quasi-static fading channels. Fig. 4 depicts the BER versus $E_{b} / N_{0}$ performance curves for RSC codes with $(1,5 / 7)$ or $(1,21 / 37)$ generator polynomials, which have memory two and four respectively, in various system settings for no iterations. Here one observes again that the effect of the coding scheme on system performance depends on the order of diversity. In particular, for SISO quasi-static fading channels different channel coding schemes do not affect the performance significantly. However as we introduce antenna diversity and increase the $E_{b} / N_{0}$, we observe that performance begins to increase as we increase the memory size over quasi-static fading channels. It was also observed that at low $E_{b} / N_{0}$ increasing the memory size actually decreased the performance of all the mapping schemes, and we define a cross-over point before which larger memory sizes are a disadvantage.

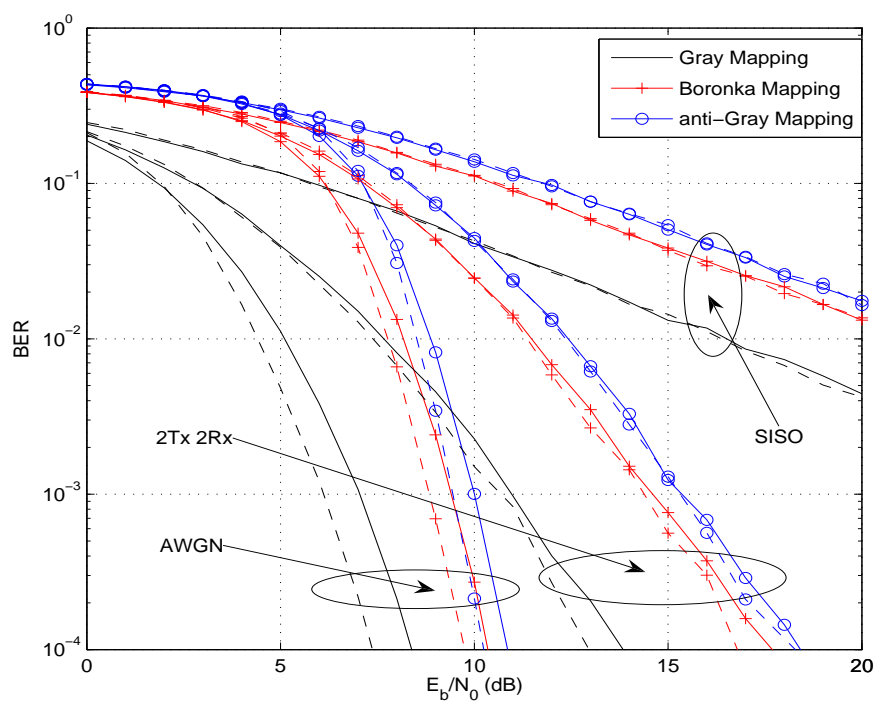

Figure 4: BER versus $E_{b} / N_{0}$ performance curves for RSC codes with $(1,5 / 7)$ (solid lines) or $(1,21 / 37)$ (dashed lines) generator polynomials over AWGN and various quasi-static channel settings, with various 16-QAM mapping schemes for no iterations. The interleaver size is 2048 . 
Again we can associate the performance over quasi-static fading channels under various different antenna diversity scenarios with sections of the AWGN channel performance. We can observe that the cross-over points under various antenna settings over quasi-static fading occur at similar BERs to those over the AWGN channel. We examine the performance in an AWGN channel and with reference to this case we postulate that at low $E_{b} / N_{0}$ values the RSC decoders are constantly being swamped by errors and the low memory codes are at an advantage since they are able to rejoin the true trellis path more quickly due to their shorter memory length. Although some of the decoded information bit may still be correct when the decoder deviates from the true path it was noted in [17] that it is intuitive that increasing the length of deviation from the true trellis would increase the bit errors. Hence we are able to consider the deviation length as a measure of the BER. In fact, we observed that when the decoding path deviated from the true trellis path the percentage of information bits that were decoded in error decreased exponentially from $100 \%$ for the minimum trellis deviation length to approximately $30 \%$ for a deviation of 20 states, whereupon it remained constant. In Fig.5 for Gray mapping we observe that when $E_{b} / N_{0}=0 \mathrm{~dB}$, increasing the memory size results in longer deviations from the true trellis path. Furthermore the cumulative dashed plot for the higher memory code surpasses the lower memory one, which will result in the observed higher BER values. At a high $E_{b} / N_{0}$ of $6 \mathrm{~dB}$, the deviation length plot is dominated by the larger quantities of shorter bursts of the lower memory code, which results in a higher cumulative dashed plot. Therefore the lower memory code achieves better BER performance at low $E_{b} / N_{0}$ values, as is depicted in Fig.4.
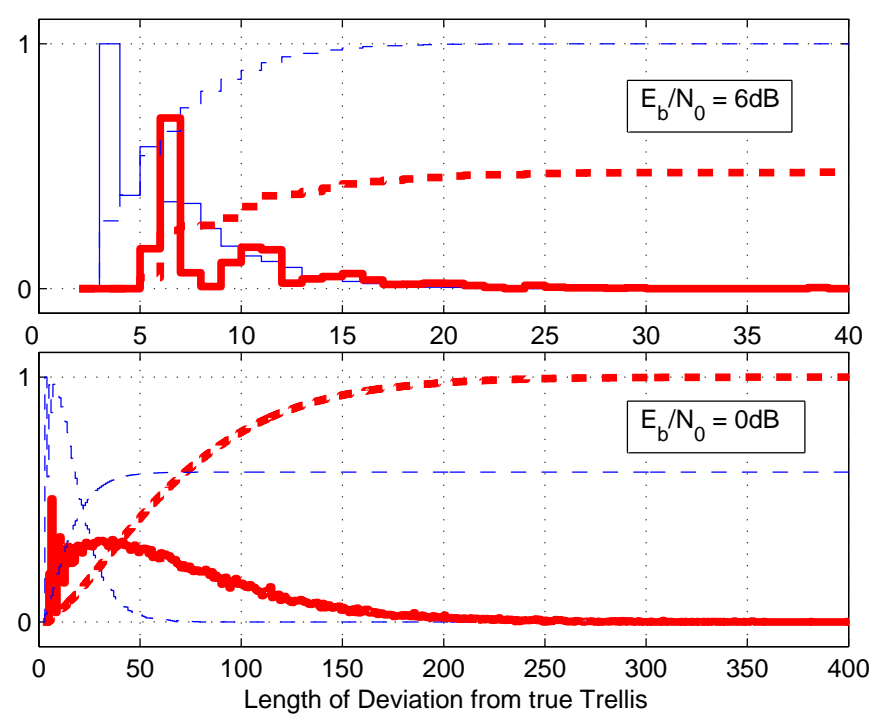

Figure 5: Normalised Histogram plot for RSC codes with (1,5/7) (thin lines) or $(1,21 / 37)$ (thick lines) generator polynomials over AWGN 16-QAM Gray mappings for no iterations. Solid Lines plot the product of path deviation from the true trellis and their frequency, giving an indication of their contribution to the BER. The frequency of occurrence is normalized by the maximum value of this product for both RSC codes, dashed Lines plot the corresponding cumulative summation normalized by the maximum summation of deviation lengths of the two RSC codes. The interleaver size is 2048 .

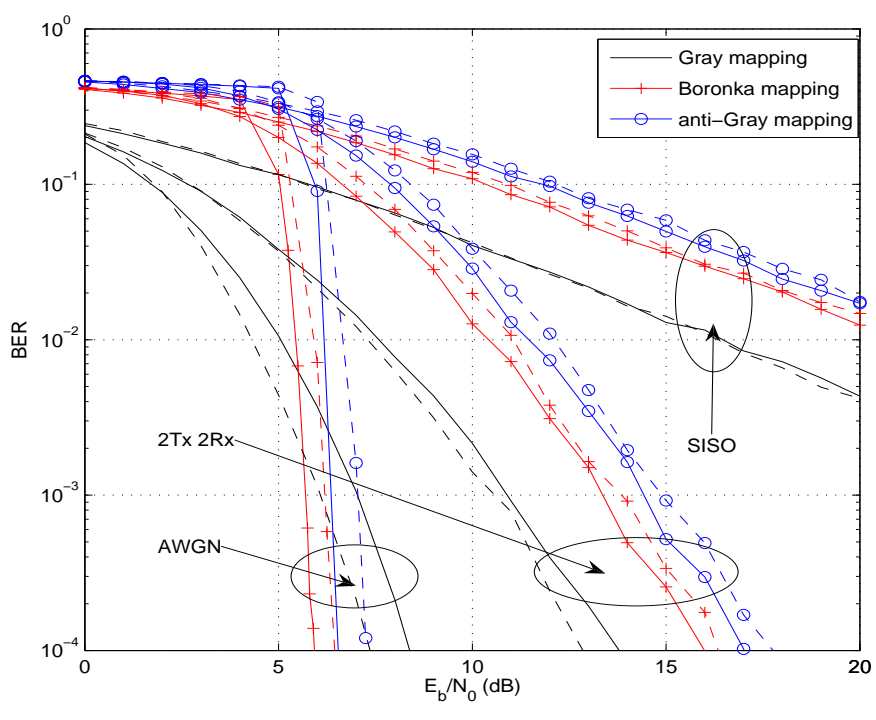

Figure 6: BER versus $E_{b} / N_{0}$ performance curves for RSC codes with $(1,5 / 7)$ (solid lines) or $(1,21 / 37)$ (dashed lines) generator polynomials over AWGN and various quasi-static channel settings, with various 16-QAM mapping schemes for the twentieth iteration. The interleaver size is 2048 .

Fig.6 shows that the systems perform very differently after 20 iterations. For both quasi-static fading and AWGN channel scenarios, increasing the memory size decreases the BER performance for anti-Gray and Boronka mappings, however Gray mapping still experiences improved performance.

\section{Effect of Interleaver - no iteration}

We believe that the pseudo-random interleaver plays an important role here as it has been observed that over a quasistatic channel with limited antenna diversity, mapping schemes optimized for iterative receivers are outperformed by mappings more appropriate for non-iterative receivers. Fig.7 shows how removing the interleaver will affect the BER performance curves for Gray, anti-Gray and Boronka 16-QAM mappings after no iterations in various system settings. We observe that removing the interleaver always improve performance for Gray mappings, whereas both anti-Gray and Boronka have a crossover point after which it becomes overwhelmingly advantageous to use interleaving.

Once more we can associate the performance over quasistatic fading channels under various different antenna diversity scenarios with sections of the AWGN channel performance. We can observe that the cross-over points under various antenna settings over quasi-static fading occur at similar BER to the AWGN channel. We examine the performance over an AWGN channel and with reference to this case we observe the reason for this cross-over. In comparison to Gray mapping Boronka has significantly larger Euclidean distances between adjacent codewords, i.e., Boronka will have a significantly larger initial error. Therefore any interleaving will break up these errors and give the decoder more chance of correctly decoding the data. These observations were borne out in the trellis deviation plots similar to Fig.5 (not included). 


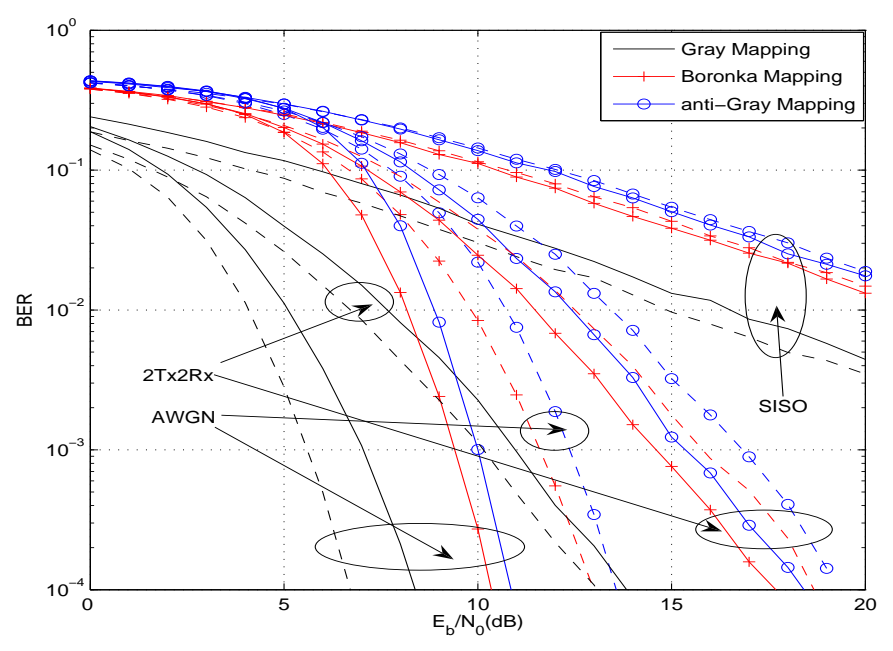

Figure 7: BER versus $E_{b} / N_{0}$ performance curves for the iterative demapping and decoding systems after no iterations based on 16-QAM mappings over AWGN and various quasi-static channel settings. The RSC code generator polynomial is $(1,5 / 7)$. The interleaver size is 2048 . Solid lines correspond to interleaving, dashed lines to no iterleaving.

\section{Effect of Interleaver Size}

Finally, we investigate the effect of the interleaver size on the performance of iterative demapping and decoding techniques over quasi-static fading channels. It is well known that the interleaver size plays a very important role in the performance of iterative schemes [1][2][5][6]. In general, the higher the interleaver size the more pronounced the "turbo cliff" [1][2][5][6]. However, we note that over quasi-static fading channels both with and without antenna diversity, different interleaver sizes result virtually in identical performance (results not shown). Again, this situation is in sharp contrast to that in the AWGN scenario.

\section{E. Final Considerations}

The previous results have demonstrated that the behavior of iterative demapping and decoding techniques in the quasi-static fading case is drastically different from that in the AWGN case. In particular, we have demonstrated that while in systems with significant diversity mappings traditionally optimized for iterative receivers (e.g., anti-Gray or Boronka mappings) outperform mappings more appropriate for non-iterative receivers (e.g., Gray mappings), in systems with limited diversity Gray based mappings in fact perform better than anti-Gray or Boronka mapping based schemes. Consequently, one concludes that the use of iterative demapping and decoding techniques (known to improve so drastically performance in an AWGN channel) is only justified over quasi-static fading channels with significant antenna diversity. Secondary results on various RSC codes and interleaving demonstrate that changes to these parameters do not affect mapping schemes to such an extent that would oppose the previous consideration, however it does affect the performance of a mapping scheme relative to itself. We observed that it is justifiable to increase the memory size and remove interleaving in quasi-static fading channels with limited antenna diversity.

\section{CONCLUSION}

In this paper, we have investigated in detail the performance of iterative demapping and decoding techniques over quasi-static fading channels both with and without antenna diversity. In particular, we have considered the effect on the system performance of various mapping schemes, different coding schemes, the interleaver, as well as space diversity. Results demonstrate that when Gray mapping is considered we should avoid the use of interleaving, both with and without antenna diversity. However when implementing iterative demapping and decoding, non-Gray mapping schemes are only suitable for quasistatic fading channels exploiting significant antenna diversity and low memory codes.

\section{REFERENCES}

[1] C. Berrou, A. Glavieux, and P. Thitimajshima, "Near Shannon limit errorcorrecting coding and decoding: Turbo-codes," in Proc. Int. Conf. Commun., vol. 2, May 1993, pp. 1064-1070.

[2] C. Berrou and A. Glavieux, "Near optimum error correcting coding and decoding: Turbo codes," in IEEE Trans. Commun., vol. 44, Oct. 1996, pp. 1261-1271.

[3] X. Wang and H. V. Poor, "Iterative (turbo) soft interference cancellation and decoding for coded CDMA," in IEEE Trans. Commun., vol. 47, July 1999, pp. 1046-1061.

[4] B. Lu and X. Wang, "Iterative receivers for multiuser space-time coding systems," in IEEE J. Select. Areas Commun., vol. 18, Nov. 2000, pp. 2322-2335.

[5] S. ten Brink, J. Speidel, and R. H. Yan, "Iterative demapping for QPSK modulation," in Electronic Letters, vol. 34, July 1998, pp. 1459-1460.

[6] - "Iterative demapping and decoding for multilevel modulation," in Proc. IEEE Globecom '98, vol. 1, July 1998, pp. 579-584.

[7] E. K. Hall and S. G. Wilson, "Design and analysis of turbo codes on Rayleigh fading channels," in IEEE J. Select. Areas Commun., vol. 16, Feb. 1998, pp. 160-174.

[8] L. Lin, L. J. Cimini, and C. I. Chuang, "Comparison of convolutional and turbo codes for OFDM with antenna diversity in high-bit-rate wireless applications," in IEEE Commun. Lett., vol. 4, Sept. 2000, pp. 277-279.

[9] H. E. Gamal and J. A. R. Hammons, "Analyzing the turbo decoder using the Gaussian approximation," in IEEE Trans. Commun., vol. 47, Feb. 2001, pp. 671-686.

[10] H. Bouzekri and S. L. Miller, "An upper bound on turbo codes performance over quasi-static fading channels," in IEEE Commun. Lett., vol. 7 , July 2003, pp. 302-304.

[11] M. R. D. Rodrigues, I. A. Chatzigeorgiou, I. J. Wassell, and R. A. Carrasco, "On the performance of turbo codes in quasi-static fading channels,' in Proc. IEEE ISIT '05, Sept. 2005, pp. 622-626.

[12] H. Bölcskei, A. J. Paulraj, K. V. S. Hari, R. U. Nabar, and W. W. Lu, "Fixed broadband wireless access: State of the art, challenges and future directions," in IEEE Commun. Mag., vol. 39, Jan. 2001, pp. 100-108.

[13] V. Tarokh, H. Jafarkhani, and A. R. Calderbank, "Space-time block codes from orthogonal designs," in IEEE Trans. Inform. Theory, vol. 45, July 1999, pp. 1456-1467.

[14] — , "Space-time block coding for wireless communications: Performance results," in IEEE J. Select. Areas Commun., vol. 17, Mar. 1999, pp. 451-460.

[15] J. Hagenauer and P. Höeher, "A Viterbi algorithm with soft-decision outputs and its applications," in Proc. IEEE Globecom '89, 1989.

[16] A. Boronka and J. Speidel, "A low complexity MIMO system based on BLAST and iterative anti-Gray-demapping," in Proc. PIMRC '03, vol. 20, Mar. 2003, pp. 284-287.

[17] L. C. Perez, J. Seghers, and D. J. Costello, Jr., "A distance spectrum interpretation of Turbo codes," in IEEE Trans. Inform. Theory, vol. 42, no. 6, Nov. 1996, pp. 1698-1709. 